\title{
PARYLENE MEMBRANE SLOT FILTER FOR THE CAPTURE, ANALYSIS AND CULTURE OF VIABLE CIRCULATING TUMOR CELLS
}

\author{
Bo Lu ${ }^{1}$, Tong $\mathrm{Xu}^{2}$, Siyang Zheng ${ }^{3}$, Amir Goldkorn ${ }^{2}$, and Yu-Chong Tai ${ }^{1}$ \\ ${ }^{1}$ California Institute of Technology, Pasadena, CA, USA \\ ${ }^{2}$ University of Southern California, Los Angeles, CA, USA \\ ${ }^{3}$ Pennsylvania State University, University Park, PA, USA
}

\begin{abstract}
This paper presents a method of capturing viable circulating tumor cells (CTC) from human whole blood using constant-pressure-driven filtration through a specially designed parylene-C membrane "slot" filter. More than $90 \%$ viable cancer cells could be recovered from whole blood using the slot filter, with minimal non-cancer blood cells left on the filter. The feasibility of the telomerase activity measurement of a single cancer cell taken from the filter after capture was proven. The on-filter and off-filter cultures of the captured cancer cells were also demonstrated.
\end{abstract}

\section{INTRODUCTION}

Cancer metastasis is still the main cause of death for patients having solid-tumor cancers. Circulating tumor cells (CTC) are tumor cells disseminated from the primary tumor into blood stream. The presence of CTC in peripheral blood has important clinical significance for patients with various types of cancers. Study of CTC will also provide valuable insight into the mechanism of tumor metastasis.

Most current CTC assays are used either for the enumeration of CTCs by immunostaining [1-7], or for specific molecular analysis by quantitative PCR (qPCR) [7,8]. For both applications, the main challenge is the extremely low concentration of CTC (e.g. $\sim 1 / \mathrm{mL}$ ). Therefore, an enrichment step of rare CTCs from a large volume (e.g. in the milliliter range) of patient blood sample is crucial for successful CTC study. So far, the reported CTC enrichment techniques include the density gradient centrifugation [7], immunomagnetic separation [7,8], microfluidic devices combined with immunoaffinity based selection [5,6] and size based filtration [1-4,7]. Size based filtration has been explored for solid-tumor cancers because the herein epithelial CTCs (15-30 $\mu \mathrm{m}$ in diameter) are generally larger and less deformable than normal blood cells. Filtration devices adopting lateral flow usually suffer from long sample processing time and limited sample volume [4]. In contrast, membrane filters have the advantage of short operation time. Single layer circular "pore" filters, including both the commercially available track-etched polycarbonate filters and microfabricated parylene filters, were reported and have been used in clinical trials $[1,7]$. However, these works mainly focused on fixed cells, which disallow further biological analysis requiring viable cells. Moreover, the required fixation or sample dilution steps of some CTC enrichment approaches [1,7] can cause CTC damage or loss.

Previously we reported two types of dual-layer 3D membrane filters [2,3] for the enrichment of viable cancer cells. They showed good capture efficiency but low enrichment factor because too many blood cells were also trapped on the filters. In this work, a specially designed single layer parylene "slot" filter is presented for viable CTC enrichment, capture and detection. To prevent the damage of CTCs, a constant-pressure-driven fluid delivery system was designed for filtration. More than $90 \%$ viable CTCs could be recovered from whole blood, with minimal non-cancer blood cells remaining on the filter. Besides enumeration, the molecular analysis of telomerase activity on a single cancer cell removed from our filter using micropipette manipulation and qPCR was also demonstrated. Moreover, the filtered cancer cells could be further cultured both on-filter and off-filter for future study.

\section{DESIGN AND FABRICATION}

Fig.1 shows the "slot" filter. Parylene-C was used as the filter material because of its good mechanical properties, machinability, biocompatibility and optical transparency in the visible range. The length of each slot was $40 \mu \mathrm{m}$, while the slot width was $6 \mu \mathrm{m}$, optimized by enumeration experiments. The fill factor was $18 \%$, which was much larger than previous "pore" filters [1].

The filter fabrication process started from depositing a $10 \mu \mathrm{m}$-thick parylene-C layer on prime silicon wafer (Fig.2). A metal layer, either $\mathrm{Cr} / \mathrm{Au}$ or $\mathrm{Al}$, was then deposited using a thermal evaporator, followed by lithography and wet-etching patterning. Parylene-C was patterned by Reactive Ion Etching (RIE) using the metal as the mask. Finally, the patterned parylene-C membrane was peeled off from the silicon substrate. 


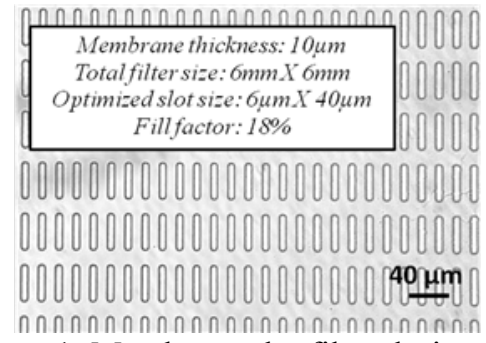

Figure 1: Membrane slot filter design

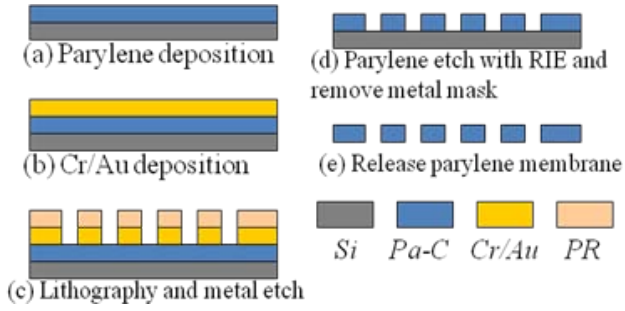

Figure 2: Fabrication process

The CTC filtration was carried out through a constant-pressure-driven fluid delivery system (Fig.3). The slot filter design and this fluid delivery system both contributed to the high viability capture. First, compared to the circular pores, slots allow easier deformation of blood cells in the slot longitudinal direction, which facilitates easier passage of normal blood cells. In addition to the slot design, the large fill factor also greatly reduces the flow resistance during filtration. Secondly, in previous filtration approaches $[1,2,7]$, samples were driven by hand-push or syringe pump. Although a near-constant flow rate could be achieved, the pressure drop $(\Delta \mathrm{P})$ across the filter increased when the filter was gradually clogged. In contrast, the constant-pressure-driven fluid delivery system is able to keep a constant $\Delta \mathrm{P}$ during filtration. The slot filter's reduced flow resistance then allows a low and constant $\Delta \mathrm{P}$ to filter out CTCs, which minimizes the forces exerted on the cells, and hence results in high viability.

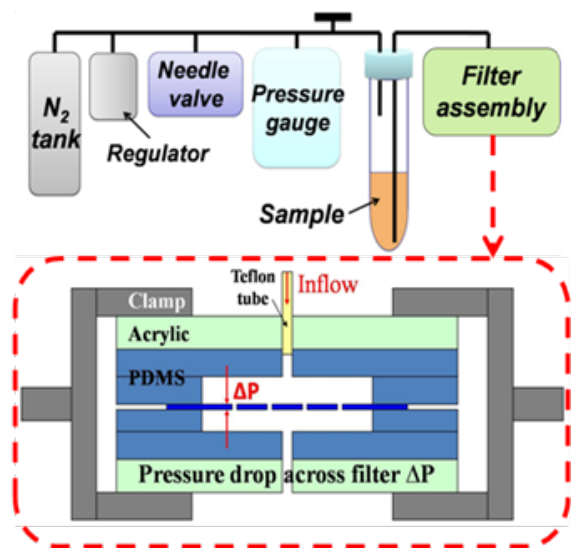

Figure 3: Constant-pressure-driven system: (a) fluid delivery system, and (b) filter assembly.

\section{EXPERIMENTAL}

Human prostate cancer PC-3 cells were cultured in RPMI 1640 culture medium supplemented with 10\% fetal bovine serum at $5 \% \mathrm{CO}_{2}$ and $37{ }^{\circ} \mathrm{C}$. For filter device testing, harvested cells were first stained with Calcein-AM fluorescent dye (Invitrogen, Carlsbad, CA, USA), centrifuged and then resuspended in phosphate buffered saline (PBS) before testing. Known number of cancer cells were spiked into $1 \mathrm{~mL}$ human whole blood obtained from a healthy donor. After filtration, captured cancer cells were counted under epifluorescence microscope. The viability of captured cancer cells was evaluated by on-filter staining with Propidium Iodide (PI) (Invitrogen). Enrichment was determined by staining white blood cells (WBCs) also captured on filter with Acridine Orange (AO) (Invitrogen) followed by WBC counting. To observe the morphology of captured cancer cells, cells on the filter went through the paraformaldehyde-glutaraldehyde fixation, multiple rinses, dehydration, air dry and sputter metal coating, and finally were viewed using a scanning electron microscope (SEM, JEOL JSM/6390LV).

To perform molecular analysis on a single captured cell, several technologies were used together. First, unlabeled PC-3 cells were spiked into $1 \mathrm{~mL}$ blood and filtrated using a slot filter. PE-conjugated anti-human CD49b antibody (BD biosciences, San Jose, CA, USA) was then used to selectively stain captured cancer cells on-filter. Next, the filter was gently washed once in PBS. Cancer cells were located under epifluorescence microscope and picked up individually from the filter through micropipette aspiration. Glass micropipettes with tip diameter of 20 $\mu \mathrm{m}$ were made by pulling glass capillaries using a P-87 micropipette puller (Sutter Instrument Company, Novato, CA, USA). The micropipette was mounted onto a XYZ stage. Targeted single cancer cell was aspirated into the micropipette by capillary force. The aspirated cell was lysed, and the telomerase assay was performed on the lysate by qPCR [9].

To prove the feasibility of the culture of the captured cancer cells, $1 \mathrm{~mL}$ whole blood spiked with unlabeled PC-3 cells was filtrated using slot filter, and the captured PC-3 cells were cultured either on-filter or off-filter. For on-filter culture, the entire filter with captured cells was soaked inside RPMI complete culture medium. For off-filter culture, captured cancer cells were first released from filter, and then cultured in a petri-dish with RPMI complete culture medium. In both cases, cells were maintained in an incubator at $5 \% \mathrm{CO}_{2}$ and $37^{\circ} \mathrm{C}$.

\section{RESULTS AND DISCUSSIONS}


Three kinds of membrane slot filters with different slot widths, $5 \mu \mathrm{m}, 6 \mu \mathrm{m}$ and $7 \mu \mathrm{m}$, were tested for their capture efficiency, viability and enrichment (Fig.4). Capture efficiency was defined as the percentage of recovered on-filter cancer cells vs. the total spiked cancer cells. Since the slot widths we studied were substantially smaller than PC-3 cancer cell size $(\sim 17$ $\mu \mathrm{m}$ in diameter), different slot widths did not cause significant variation of capture efficiency (Fig.4a). Cancer cell viability was the percentage of live cancer cells captured on filter, evaluated by both Calcein-AM and PI staining (Fig.5a\&b). Viable cancer cells with intact cell membrane were able to retain Calcein-AM, thus fluoresced green brightly. Dead cancer cells with compromised cell membrane were stained by red-fluorescent nuclear dye PI, while the green fluorescence became faint due to Calcein-AM dye leakage. A remarkable drop of viability was observed when the slot width increased to $7 \mu \mathrm{m}$ (Fig.4a). Under flow pressure and shear stress, captured cancer cells were forced to stretch and partially deform into the slot if the slot was too wide. In this condition, the rupture of cell membrane and the internal damage of the cell were possible reasons for the drop of viability. A noticeable reduction of capture efficiency can be predicted if the slot width further increases and cancer cells are able to deform through the slot. Enrichment was defined as the ratio of WBCs remaining on filter to the total amount of WBCs. A clear discrepancy appeared between $5 \mu \mathrm{m}$ and $6 \mu \mathrm{m}$ slot widths (Fig.4b). This difference was probably due to some large monocytes $(12-20 \mu \mathrm{m})$ [1], which may have threshold passage width between $5 \mu \mathrm{m}$ and $6 \mu \mathrm{m}$. Based on above analysis, the filter with $6 \mu \mathrm{m}$ slot width was the optimal design since it had over $90 \%$ capture efficiency and viability, with only $0.48 \%$ WBCs and minimal amount RBCs left on filter. Filter performance under different drive pressures was also examined. As drive pressure increased, both capture efficiency and viability decreased (Fig.4c). Hence low drive pressure was critical for live cell capture. Experimentally, 0.13 psi was found as the optimal drive pressure for high viability and successful sample processing. Filtration of $1 \mathrm{~mL}$ sample under the optimal conditions took less than 5 minutes. As so, the morphology of captured cancer cells remained unharmed as indicated by SEM (Fig.5c).

Compared to normal cells, most cancer cells have higher telomerase activity, which directly indicates their malignant/metastatic potential $[8,9]$. Hence, the measurement of telomerase activity of CTCs is important for cancer metastasis study. However, there are difficulties. First, telomerase activity can only be measured from viable cells. Secondly, although the telomerase activity of individual WBC is low, without an efficient enrichment, the telomerase activity from all the WBCs may still add high background noise to the telomerase activity of CTCs. By picking up a single cancer cell using micropipette from the enriched viable cancer cells on the filter, we could isolate the single cancer cell and obtain its telomerase activity by sensitive qPCR. Fig. 6 shows the measured telomerase activity of both recovered cancer cells and control cells. Different $\mathrm{Ct}$ values (threshold cycle) mean the telomerase activity varied among different cells. Higher telomerase activity (lower $\mathrm{Ct}$ ) is associated with a more aggressive, metastatic cancer phenotype.
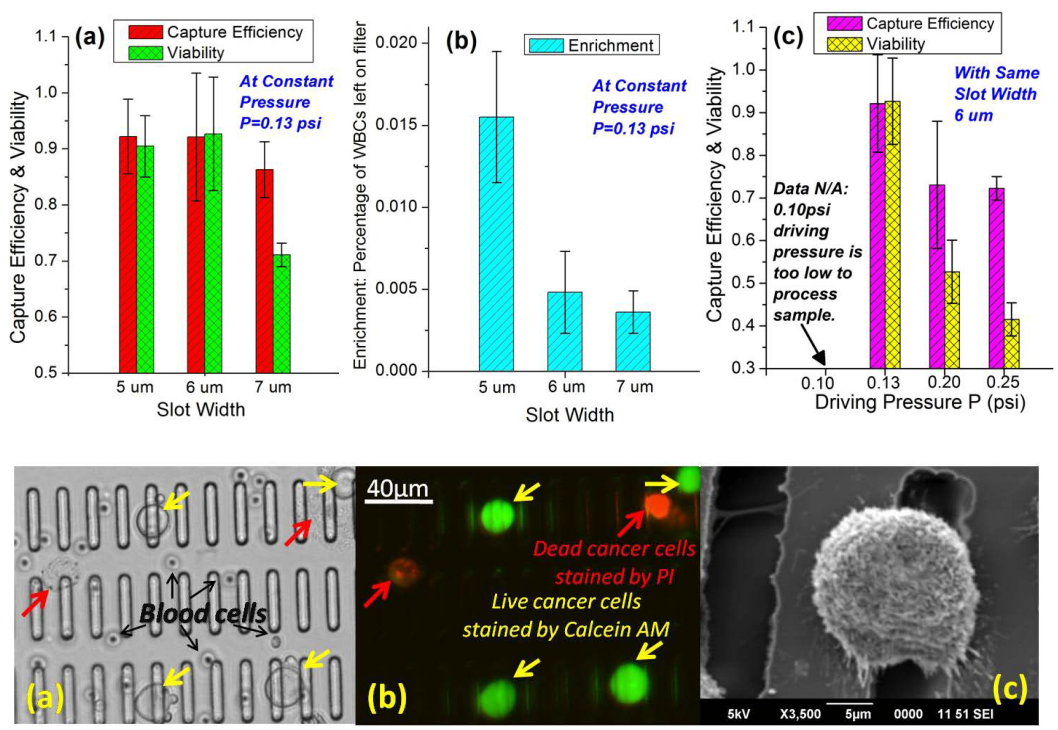

Figure 4: Filter characterization: (a)(b) Capture efficiency, viability and enrichment with slot size at the same drive pressure of $0.13 \mathrm{psi}$; (c) Capture efficiency and viability with drive pressure with the same slot width of $6 \mu \mathrm{m}$.

Figure 5: Cancer cells captured by filter from $1 \mathrm{~mL}$ whole blood. (a)(b) Bright field and merged fluorescent pictures taken at same spot. Live cells (green) stained with Calcein-AM; dead cells (red) stained with PI. (c) SEM picture of captured cancer cell.
The culture of captured CTCs is highly desirable for further studies of the cancer cells. However, most previously published methods had the problems of low viability, lack of biocompatible environment or incapability to release captured cells $[1,2,5-7]$. Here, the slot filter was demonstrated to have overcome 
these problems, for both on-filter and off-filter culture. For on-filter culture, even without any surface pre-treatment, PC-3 cells were able to adhere and proliferate on the filter surface (Fig.7a). For off-filter culture, trapped cells were first released and collected from the filter, by multiple rinses or reverse flow (passing PBS from backside), and then cultured in petri-dish (Fig.7b).
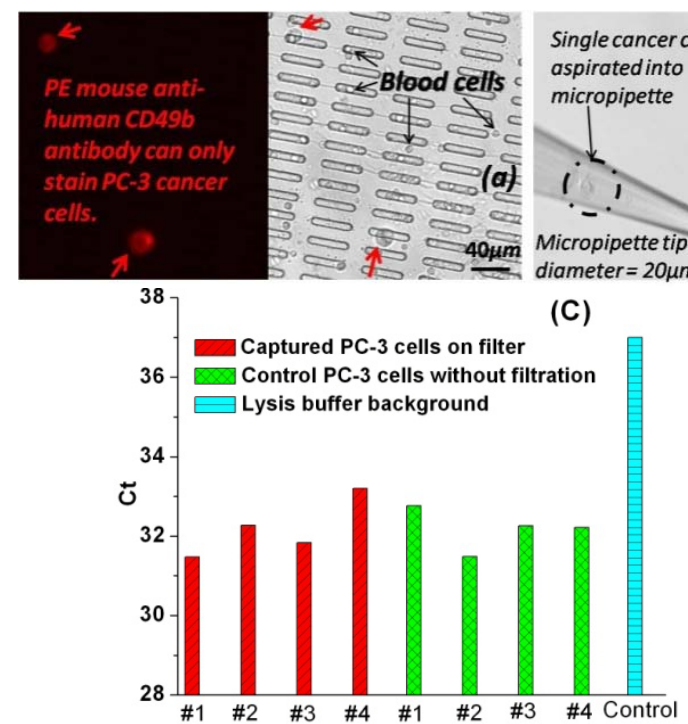

Figure 6: Single cell telomerase measurement. (a) Use on-filter immunostaining to find the locations of cancer cells (red arrows: cancer cells; black arrows: blood cells); (b) Single cancer cell was picked up by micropipette aspiration; (c) Single cancer cell telomerase activity measured by qPCR.

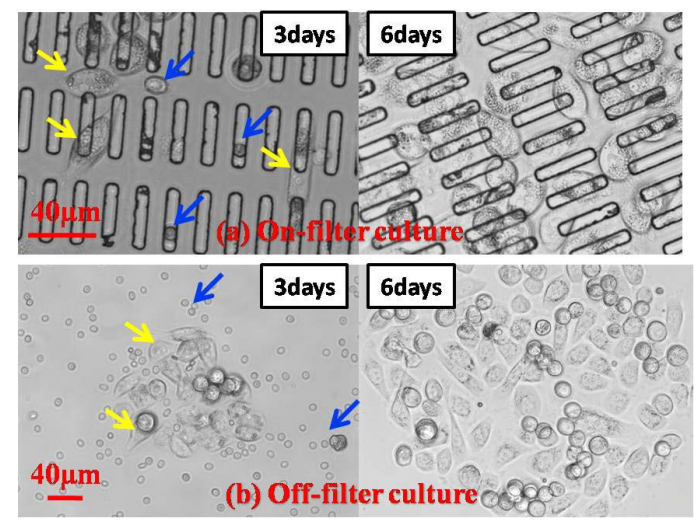

Figure 7: On-filter (a) and off-filter (b) cell culture of captured PC-3 cells from human blood after 3 days and 6 days in RPMI complete medium. Yellow arrows show examples of cancer cells spreading and dividing, and blue arrows show examples of remaining blood cells after filtration.

\section{CONCLUSION}

A parylene membrane slot filter and a constant-pressure-driven fluid delivery system were designed for the enrichment, capture and detection of viable CTCs from whole blood. More than $90 \%$ viable cancer cells were captured, with minimal amount of non-cancer blood cells remaining on filter. The feasibility of the telomerase activity measurement of a single cancer cell taken from the filter after filtration was proven. The on-filter and off-filter cultures of the captured cancer cells were also demonstrated.

\section{ACKNOWLEDGEMENT}

The funding of this project is provided by NIH/NCI 1R01CA141077-01, and The Hope Foundation SWOG0421.

\section{REFERENCES}

[1] S. Zheng, H. Lin, J. Q. Liu, M. Balic, R. Datar, R. J. Cote and Y. -C. Tai, "Membrane microfilter device for selective capture, electrolysis and genomic analysis of human circulating tumor cells", Journal of Chromatography A, vol. 1162, pp. 154-161, 2007.

[2] S. Zheng, H. Lin, R. J. Cote and Y. -C. Tai, “A novel 3D micro membrane filtration device for capture viable rare circulating tumor cells from whole blood", Proc. of Hilton Head 2008, Hilton Head Island, SC, USA, 2008, pp. 134-137.

[3] B. Lu, S. Zheng, S. Xie and Y. -C. Tai, "Live capture of circulating tumor cells from human blood by a splitable $3 \mathrm{D}$ parylene membrane filtration device", Proc. of $\mu$ TAS 2009, Jeju, Korea, 2009, pp. 588-590.

[4] S. J. Tan, L. Yobas, G. Y. H. Lee, C. N. Ong and C. T. Lim, "Microdevice for the isolation and enumeration of cancer cells from blood", Biomedical Microdevices, vol. 11, pp. 883-892, 2009.

[5] S. Nagrath, L. V. Sequist, S. Maheswaran, et al. "Isolation of rare circulating tumor cells in cancer patients by microchip technology", Nature, vol. 450, pp. 1235-1239, 2007.

[6] A. P. Horton, K. Kumar, H. Liu, et al. "Quantitative immunophenotyping of circulating tumor cells in blood using microfluidic screening chip integrated with hyperspectral microscope", Proc. of Transducers 2009, Denver, CO, USA, 2009, pp. 77-80.

[7] P. Paterlini-Brechot and N. L. Benali, "Circulating tumor cells (CTC) detection: Clinical impact and future directions", Cancer Letters, vol. 253, pp. 180-204, 2007.

[8] K. Fizazi, L. Morat, L. Chauveinc, et al. "High detection rate of circulating tumor cells in blood of patients with prostate cancer using telomerase activity" Annals of Oncology, vol. 18, pp. 518-521, 2007.

[9] B. S. Herbert, A. E. Hochreiter, W. E. Wright, et al. "Nonradioactive detection of telomerase activity using the telomeric repeat amplification protocol", Nature Protocols, vol. 1, pp. 1583-1590, 2006. 